\title{
BCR-NTRK2 fusion in a Pediatric Patient with Spinal Gangliocytoma Avanti Gupte, M.D.1, Neena I. Marupudi, M.D.1,2, M.S. 1,Swati Mody, M.D
}

Avanti Gupte $^{1}$, Neena Marupudi ${ }^{1}$, Swati Mody ${ }^{1}$, William Kupsky ${ }^{1}$, and Hamza Gorsi ${ }^{1}$

${ }^{1}$ Children's Hospital of Michigan

March 10, 2021

\section{BCR-NTRK2 fusion in a Pediatric Patient with Spinal Gangliocytoma}

Avanti Gupte, M.D. ${ }^{1}$, Neena I. Marupudi, M.D. ${ }^{1,2}$, M.S. ${ }^{1}$,Swati Mody, M.D. ${ }^{1,2}$, William Kupsky M.D. ${ }^{1,2}$, Hamza S. Gorsi, M.D., M.P.H. ${ }^{1,3}$

\section{Affiliations:}

${ }^{1}$ Children's Hospital of Michigan/Detroit Medical Center, Michigan, U.S.A

${ }^{2}$ Wayne State University, Michigan, U.S.A

${ }^{3}$ Central Michigan University, U.S.A

\section{Address Correspondence to:}

Avanti Gupte, Department of Pediatric Hematology Oncology, Children's Hospital of Michigan, 3901 Beaubien St, Detroit MI 48201, (agupte@dmc.org ), 313-745-5515

Hamza Gorsi, Department of Pediatric Hematology Oncology, Children's Hospital of Michigan, 3901 Beaubien St, Detroit MI 48201, (hgorsi@dmc.org), 313-745-5515

Word Count: [712 words]

Number of Figures: 1

Short Title: BCR-NTRK2 mutation in pediatric gangliocytoma

Keywords: Gangliocytoma, BCR-NTRK2, BRAF, Trk

\section{Abbreviations:}

\begin{tabular}{ll}
\hline Abbreviation & Full Term \\
\hline WHO & World Health Organisation \\
BCR & Breakpoint Cluster Region \\
NTRK2 & Neurotrophic Receptor Tyrosine Kinase 2 \\
MRI & Magnetic Resonance Imaging \\
Trk & Tropomyosin Receptor Kinase \\
NGF & Nerve Growth Factor \\
BDGF & Brain Derived Growth Factor \\
NT3 & Neurotrophin-3 \\
FDA & Food and Drug Administration \\
\hline
\end{tabular}




\section{To the editor,}

Gangliocytomas are WHO grade I neuroepithelial tumors consisting of mature ganglion cells intermixed with normal glial cells (1). They usually present between 10-30 years of age and comprise less than $0.5 \%$ of the CNS tumors (1). The most common sites for presentation are temporal lobe, cerebral hemispheres, cerebellum (Lhermitte-Duclos disease), floor of the $3^{\text {rd }}$ ventricle and spinal cord (1). Spinal gangliocytomas are exceedingly rare, comprising less than $10 \%$ of all gangliocytomas (1). Maximal safe resection is the mainstay of treatment, as this tumor does not respond to irradiation or traditional chemotherapy (1). Very little is known about the molecular makeup or the role of targeted therapy in this tumor. We present a novel molecular finding, BCR-NTRK2 fusion and overexpression of BRAF, in a pediatric patient with spinal gangliocytoma.

A 7-year-old male child with no significant past medical history presented with scoliosis, prompting work-up with magnetic resonance imaging (MRI) of the spine. He had no back pain or neurologic deficits. MRI of the spine revealed a heterogeneously enhancing, expansile solid and cystic intradural, intramedullary, T2 hyperintense infiltrating mass extending from T6 to L2 with associated cranial and caudal syrinx formation (Fig 1). Patient underwent T6-T12 laminectomy and near total resection of the intramedullary intradural spinal cord tumor. Postoperatively, patient had mild sensory and proprioception deficits that resolved within a few weeks. Histopathology demonstrated moderately cellular, abnormal neural tissue composed of irregular clusters and cords of mature appearing tumor neurons in a coarse neutrophil background, without microvascular proliferation or necrosis, diagnostic of gangliocytoma of the spinal cord (Fig 1). MRI brain did not show any intracranial mass. Patient remains neurologically stable and has been monitored with serial MRIs of the spine over 2 years with no growth or new recurrence of tumor (Fig 1). Molecular sequencing of the tissue demonstrated somatic BCR-NTRK2 fusion and overexpression of BRAF. No germ line mutations were noted.

Gangliocytomas are not radiosensitive or chemo sensitive tumors; therefore, the goals of surgical treatment gross total or maximal safe resection in order to optimize long-term outcomes. Molecular analysis was performed to identify any targetable gene mutations for future therapy, revealing a BCR-NTRK2 fusion. This mutation has not previously been reported in gangliocytomas.

NTRK fusion genes have been reported in a number of adult and pediatric malignancies. They are reported with high frequency in rare adult malignancies like secretory breast carcinoma, mammary analog secretory carcinoma (MASC) and with low frequency in common adult malignancies like breast, lung, colorectal cancers, melanoma, ALL and AML (2). NTRK2 fusions have also been described in adult low grade gliomas (2). In pediatric malignancies, these fusions are fairly common in infantile fibrosarcoma, congenital mesoblastic nephroma, papillary thyroid cancer and soft tissue sarcomas/mesenchymal tumors (2). NTRK fusions have also been reported in non-brainstem high grade gliomas in infants (2).

Tropomyosin receptor kinase (Trk) family of receptors Trk A, B and C are encoded by NTRK1, NTRK2 and NTRK3 genes respectively (3). Ras-ERK, PI3K-AKT and PLC are the three pathways involved in TRK signaling. The ligand NGF (nerve growth factor) binds to TrkA, BDGF (brain derived growth factor) binds to Trk B and NT3 (neurotrophin-3) binds with TrkC but also with lower affinity to the other two receptors. (3). Binding of the respective ligands with TrkA and B receptors activates all three pathways while TrkC activates the Pi3-AKT pathway (3). The activation of these pathways is responsible for cell differentiation and proliferation (3). The genetic fusions involving NTRK1, 2 and 3 genes result in constitutively active or overexpressed receptor tyrosine kinases, resulting in oncogenesis (3).

Larotrectinib received FDA approval in November 2018 for a wide array of pediatric and adult solid tumors with NTRK fusions. Entrectinib is another pan Trk inhibitor with activity against ROS1 and ALK (2) as well. In the event of future disease progression, molecularly targeted therapy with Larotrectinib or Entrectinib may be an option for this particular patient..

Molecular testing of rare tumors like spinal gangliocytomas provides insights into oncologic transformation, allowing for a precision medicine approach to treatment. Viable molecularly targetable treatment options 
can be identified and implemented at the time of recurrence or progression in low-grade tumors like gangliocytoma, potentially minimizing the need for repeat surgical resection when traditional treatment options like chemotherapy or radiation have been exhausted or are not applicable.

Funding Source : No funding was secured for this study.

Conflicts of interest : The authors have no conflicts of interest to disclose.

Author's contribution : All authors contributed significantly to this manuscript, reviewed and agreed upon its content.

\section{REFERENCES:}

1: Jacob JT, Cohen-Gadol AA, Scheithauer BW, Krauss WE. Intramedullary spinal cord gangliocytoma: case report and a review of the literature. Neurosurg Rev. 2005 Oct;28(4):326-9. doi: 10.1007/s10143-0050383-8. Epub 2005 Mar 1. PMID: 15739067.

doi: 10.1007/s002470100445. PMID: 11373931.

2: Cocco E, Scaltriti M, Drilon A. NTRK fusion-positive cancers and TRK inhibitor therapy. Nat Rev Clin Oncol. 2018 Dec;15(12):731-747. doi: 10.1038/s41571-018-0113-0. PMID: 30333516; PMCID: PMC6419506.

3: Gatalica Z, Xiu J, Swensen J, Vranic S. Molecular characterization of cancers with NTRK gene fusions. Mod Pathol. 2019 Jan;32(1):147-153. doi: 10.1038/s41379-018-0118-3. Epub 2018 Aug 31. PMID: 30171197.

\section{Legends:}

Figure 1: A: T2 weighted image at diagnosis. B: Post contrast T1 weighted image at diagnosis. C: T2 weighted image post resection. D: Post contrast T1 weighted image post resection. E:Disorganised array of NeuN-immunoreactive mature-appearing neurons. F: NFP highlights dense background of axons and occasional neuronal cell bodies. G:Few scattered reactive astrocytes (neurons are unstained).
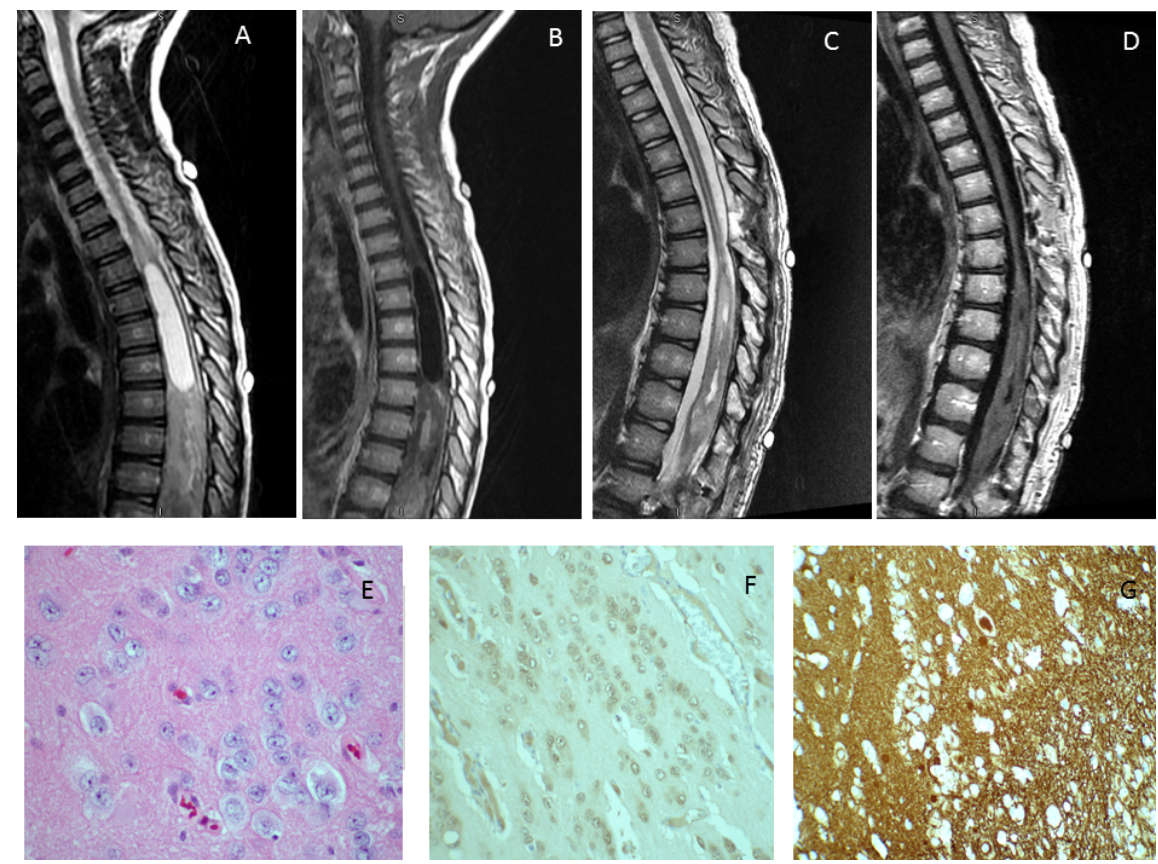